A2

doi: 10.14232/ syrpharmacognosy.2021.a2

\title{
Studies on oxidized resveratrol metabolite mixtures
}

\author{
Orinamhe Godwin Agbadua
}

Email: orinamhe.agbadua@pharmacognosy.hu

Resveratrol is a well-known polyphenol with a plethora of pharmacological activities [1,2]. Due to its chemical structure, resveratrol may directly scavenge reactive oxygen and nitrogen species. In living systems, this can lead to the generation of a wide range of new metabolites with altered bioactivities [3].

The aim of our studies is to evaluate the potential biological relevance of this phenomenon. The oxidation of resveratrol through various chemical reactions, including biomimetic approaches, resulted in mixtures that exhibited greater bioactivities activities compared to the parent compound. Mixtures were tested for in vitro DPPH and ORAC antioxidant activities, and inhibitory action on lipoxygenase, xanthine oxidase, acetylcholinesterase and angiotensin converting enzymes. Based on chromatographic fingerprint and chemical diversity, promising mixtures were selected for activity-guided isolation.

To this end, isolation of constituents from the bioactive oxidized resveratrol mixtures afforded 31 compounds in pure form, which are currently being investigated for antioxidant and enzyme inhibitory bioactivities. Their structure elucidation is mostly ongoing, and several resveratrol dimers are assumed based on preliminary data.

In conclusion, the oxidation of resveratrol led to the formation of new metabolites that showed greater inhibitory activities to several enzymes of significant clinical importance when compared to the parent compound.

Supervisor: Attila Hunyadi

\section{Acknowledgements:}

This work was supported by the National Research, Development and Innovation Office, Hungary (NKFIH; K-134704), the Economic Development and Innovation Operative Program GINOP-2.3.2-15-2016-00012, and the Ministry of Human Capacities, Hungary grant 20391-3/2018/FEKUSTRAT. I would also like to acknowledge the Stipendium Hungaricum Scholarship.

\section{References}

[1] Shaito A et al. International Journal of Molecular Sciences 2020; 21:2084

[2] Duarte A et al Food and Science Technology 2015; 63:1254-1260.

[3] Hunyadi A Medical Research Reviews 2019; 1-29. 